\title{
Design of Asymmetrical Relay Resonators for Maximum Efficiency of Wireless Power Transfer
}

\author{
Bo-Hee Choi and Jeong-Hae Lee \\ Department of Electronic Information and Communication Engineering, Hongik University, Seoul 121-791, Republic of Korea \\ Correspondence should be addressed to Jeong-Hae Lee; jeonglee@hongik.ac.kr
}

Received 28 December 2015; Revised 15 March 2016; Accepted 29 March 2016

Academic Editor: Francisco Falcone

Copyright ( 2016 B.-H. Choi and J.-H. Lee. This is an open access article distributed under the Creative Commons Attribution License, which permits unrestricted use, distribution, and reproduction in any medium, provided the original work is properly cited.

\begin{abstract}
This paper presents a new design method of asymmetrical relay resonators for maximum wireless power transfer. A new design method for relay resonators is demanded because maximum power transfer efficiency (PTE) is not obtained at the resonant frequency of unit resonator. The maximum PTE for relay resonators is obtained at the different resonances of unit resonator. The optimum design of asymmetrical relay is conducted by both the optimum placement and the optimum capacitance of resonators. The optimum placement is found by scanning the positions of the relays and optimum capacitance can be found by using genetic algorithm (GA). The PTEs are enhanced when capacitance is optimally designed by GA according to the position of relays, respectively, and then maximum efficiency is obtained at the optimum placement of relays. The capacitance of the second resonator to $n$th resonator and the load resistance should be determined for maximum efficiency while the capacitance of the first resonator and the source resistance are obtained for the impedance matching. The simulated and measured results are in good agreement.
\end{abstract}

\section{Introduction}

Wireless power transfer (WPT) is very useful and applicable technology in many areas, for example, smart phone [1], smart car [2], home appliances, medical devices [3-6], automated logistics, and robots such as drone. In particular, WPT will be more needed to apply to Internet of things (IoT) and wearable devices in the near future. So far, induction method WPT application has been actively commercialized, for example, charging of smart phone, charging of electric toothbrush, and powering of automated logistics, but magnetic resonance WPT could hardly be commercialized and is currently being developed. However, magnetic resonance WPT application will be actively developed and commercialized since The Alliance for Wireless Power (A4WP) and Power Matters Alliance (PMA) are consolidated in June 2015 which are standards of magnetic and induction resonance WPT, respectively [7].

Magnetic resonance WPT [8] has an advantage compared with induction WPT. Magnetic resonance WPT can transfer power to longer distance of tens of centimeters effectively by using the resonance of a transmitter and a receiver whereas the inductive coupling method is used in short distance of many millimeters. However, magnetic resonance WPT cannot prevent the efficiency from dropping gradually as the distance between a transmitter and a receiver is longer [9]. Therefore, the researches to improve PTE have been conducted actively. The researches of an optimum load, a metamaterial slab, and a frequency-tuning method are demonstrated [10-15].

Another approach to improve the power transfer efficiency at long distance is to employ the relay resonators [1627]. Many researches on relay resonators of magnetic resonance WPT have been conducted to extend the distance of power transfer. By adding relay resonators, PTE is improved at long distance between transmitting and receiving resonator [16]. The optimum position of one relay between transmitting and receiving resonators was investigated [17] and number of relays and distance between relays were optimized for 
maximum PTE [18]. In addition, it is possible to control power flow by employing relay resonators $[19,20]$.

The analysis of PTE in a relay resonator system was complicated. In [21, 22], nonadjacent coupling of relays was ignored because nonadjacent coupling is smaller than adjacent coupling. However, the analysis ignoring nonadjacent coupling could not give birth to an exact result. The research of relay resonators by considering nonadjacent coupling was conducted [23]. It has been proved that the maximum PTE is not achieved at the resonance frequency of unit resonator due to magnetic couplings between nonadjacent resonators [23]. Therefore, it is clear that a new design method of relay resonators is required to obtain the maximum PTE. The design method of optimization of capacitance of each resonator was employed in case of symmetric relay configuration [24] and the optimum placement of the asymmetrical relay resonators was found as the positions of the relays are scanned [25]. However, the PTE of the asymmetrical relay resonators is not maximized because the capacitance of each resonator is not designed optimally.

In this paper, the optimum design of asymmetrical relay resonator is presented by optimizing the capacitance of each resonator as well as the placement of asymmetrical relay resonator. Additional improvement of PTE is achieved using the optimum capacitance determined by both PTE equation and GA after optimizing the placement of resonator. Finally, the maximum power transfer efficiency can be obtained by finding both the optimum positions and the optimum capacitance of the asymmetrical relay resonators.

\section{Equivalent Circuit of Asymmetrical Relay Resonators}

Figure 1 shows a structure of asymmetrical relay resonators. There are four resonators: a transmitter, two relays, and a receiver. The distance between a transmitter and a receiver is fixed to be $75 \mathrm{~cm}$. The 2 relays are added between a transmitter and a receiver. The 2 relays can be rearranged between a transmitter and a receiver.

Figure 2 shows the equivalent circuit of asymmetrical relay resonators with a source and a load. $R_{i}, L_{i}$, and $C_{i}$ $(i=1,2, \ldots, n)$ are the resistance, self-inductance, and lumped capacitance connected in series to the $i$ th resonator, respectively. $M_{i j}(i, j=1,2, \ldots, n, i \neq j)$ is the mutual inductance between $i$ th resonator and $j$ th resonator. $R_{S}$ and $R_{L}$ are source and load resistance, respectively. $I_{i}(i=$ $1,2, \ldots, n)$ is the current flowing on the $i$ th resonator. $V_{s}$ is source voltage and $Z_{\text {in }}$ is input impedance. It is noted that $R_{i}, L_{i}$, and $M_{i j}$ are constants because the size of resonators and distance are fixed. Therefore, the design variables become $C_{1}, \ldots, C_{n}, R_{S}$, and $R_{L}$. The purpose of this work is to find the optimum design variables for maximum power transfer of asymmetrical relay resonators.

Kirchhoff's voltage law (KVL) equations of the equivalent circuit of Figure 2 are given in the following matrix form:

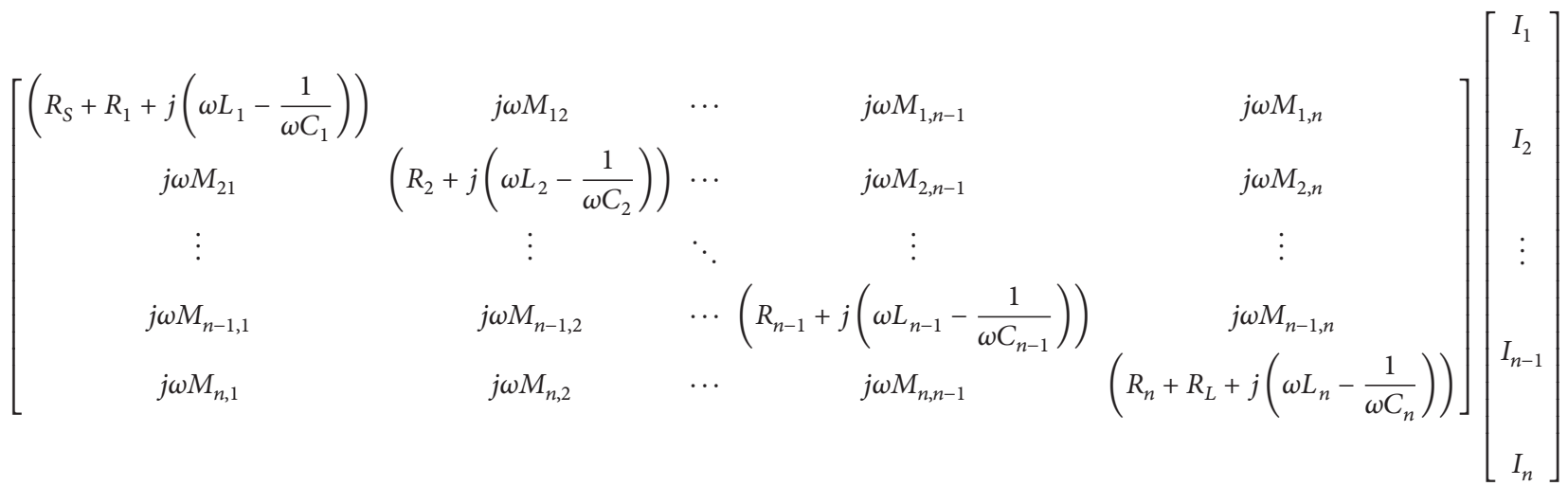

$$
\begin{aligned}
& =\left[\begin{array}{c}
V_{S} \\
\\
0 \\
\vdots \\
0 \\
\\
0
\end{array}\right] \text {, }
\end{aligned}
$$

where $\omega$ is an angular frequency. 


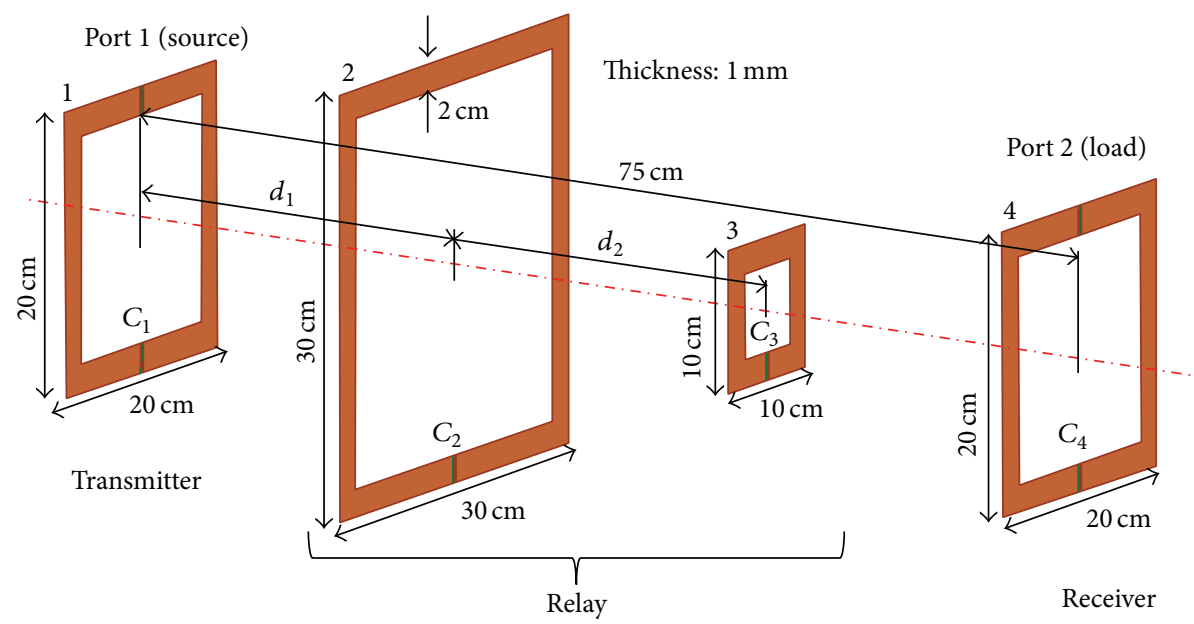

FIgURE 1: Structure of the asymmetrical relay resonators.

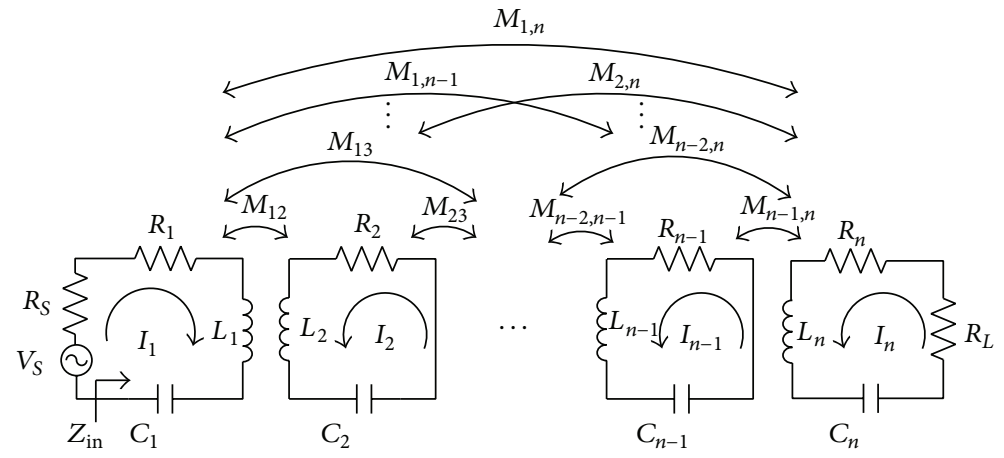

FIGURE 2: Equivalent circuit of asymmetrical relay resonators.

The efficiency of wireless power transfer is defined as the ratio of the dissipated power $\left(P_{L}\right)$ at the load to the input power $\left(P_{\text {in }}\right)$ which can be obtained by adding the total dissipated power in the resonators and the load. The PTE is given by [24]:

$$
\begin{aligned}
\eta & =\frac{P_{L}}{P_{\text {in }}}=\frac{(1 / 2) R_{L}\left|I_{n}\right|^{2}}{(1 / 2) R_{1}\left|I_{1}\right|^{2}+(1 / 2) R_{2}\left|I_{2}\right|^{2}+\cdots+(1 / 2) R_{n-1}\left|I_{n-1}\right|^{2}+(1 / 2)\left(R_{n}+R_{L}\right)\left|I_{n}\right|^{2}} \\
& =\frac{R_{L}}{R_{1}\left|I_{1} / I_{n}\right|^{2}+R_{2}\left|I_{2} / I_{n}\right|^{2}+\cdots+R_{n-1}\left|I_{n-1} / I_{n}\right|^{2}+\left(R_{n}+R_{L}\right)} .
\end{aligned}
$$

To calculate the efficiency of (2), the current ratio should be obtained from (1). The current column $\left[I_{1} \cdots I_{n}\right]^{T}$ of (1) should be normalized by $I_{n}$. Then, the first row $\left[\left(R_{S}+R_{L}+\right.\right.$ $\left.\left.j\left(\omega L_{1}-1 / \omega C_{1}\right)\right) \cdots j \omega M_{1, n}\right]$ and $\left[V_{s}\right]$ of (1) can be erased and the last column $\left[j \omega M_{2, n} \cdots\left(R_{n}+R_{L}+j\left(\omega L_{n}-1 / \omega C_{n}\right)\right)\right]^{T}$ can be transposed to the right hand side. Therefore, the last row of the current matrix $\left[I_{n} / I_{n}\right]$ is erased. Then, it becomes as follows: 


$$
\begin{gathered}
{\left[\begin{array}{cccc}
j \omega M_{21} & \left(R_{2}+j\left(\omega L_{2}-\frac{1}{\omega C_{2}}\right)\right) & \ldots & j \omega M_{2, n-1} \\
\vdots & \ddots & \vdots \\
j \omega M_{n-1,1} & j \omega M_{n-1,2} & \ldots & \left(R_{n-1}+j\left(\omega L_{n-1}-\frac{1}{\omega C_{n-1}}\right)\right) \\
j \omega M_{n, 1} & j \omega M_{n, 2} & \ldots & j \omega M_{n, n-1}
\end{array}\right]\left[\begin{array}{c}
\frac{I_{1}}{I_{n}} \\
\frac{I_{2}}{I_{n}} \\
\vdots \\
\frac{I_{n-1}}{I_{n}}
\end{array}\right]} \\
\left.=\left[\begin{array}{c}
-j \omega M_{2, n} \\
\vdots \\
-j \omega M_{n-1, n} \\
-\left(R_{n}+R_{L}+j\left(\omega L_{n}-\frac{1}{\omega C_{n}}\right)\right.
\end{array}\right]\right)
\end{gathered}
$$

From (3), the current ratio $I_{1} / I_{n}, \ldots$, and $I_{n-1} / I_{n}$ can be obtained by using inverse matrix and they are substituted for (2). Therefore, the PTE $(\eta)$ becomes a function of $C_{2}, \ldots, C_{n}$ and $R_{L}$ as follows:

$$
\eta=f\left(C_{2}, \ldots, C_{n}, R_{L}\right) .
$$

Note that $C_{1}$ and $R_{S}$ are not related to PTE $(\eta)$ but are related to impedance matching. Our purpose is to determine $C_{2}, \ldots, C_{n}$ and $R_{L}$ for maximum PTE. $C_{1}$ and $R_{S}$ are also important for impedance matching that will be determined by impedance matching condition.

\section{Optimum Design of Asymmetrical Relay Resonators}

First, the optimum resistance of $R_{L, \text { opt }}$ should be expressed as a function of $C_{2}, C_{3}, \ldots$, and $C_{n-1}$ to satisfy the equation of $\partial \eta\left(C_{2}, \ldots, C_{n}, R_{L}\right) / \partial R_{L}=0$ [17]. The equation of $R_{L, \text { opt }}=$ $f\left(C_{2}, C_{3}, \ldots, C_{n-1}\right)$ is substituted to (4) and, then, PTE $(\eta)$ becomes a function of $C_{2}, C_{3}, \ldots$, and $C_{n}$. To obtain the optimum capacitance of $C_{2, \mathrm{opt}}, C_{3, \mathrm{opt}}, \ldots$, and $C_{n \text {,opt }}$ for maximum efficiency, the equations of $\partial \eta / \partial C_{2}=0, \ldots$, and $\partial \eta / \partial C_{n}=0$ should be simultaneously solved but they are very complicated. Therefore, $C_{2, \mathrm{opt}}, \ldots$, and $C_{n, \mathrm{opt}}$ could be determined by GA and PTE equation of (4). GA is a search heuristic that mimics the process of natural selection. It used to generate useful solution to optimization and search problems. GA finds the minimum value of fitness function by repetition of selection, crossover, and mutation which are inherent process $[24,28]$. This GA procedure is summarized in Figure 3 and GA options are listed in Table 1. The minimum value of fitness function, that is, negative PTE equation of (4), is found through the repetition of selection, crossover, and mutation which are inherent process of GA. Finally, the minimum value of negative PTE corresponding to maximum PTE is found when the optimum capacitance is determined. The optimization algorithm of GA was implemented by MATLAB code. After $C_{2, \mathrm{opt}}, \ldots$, and $C_{n, \mathrm{opt}}$ are determined, $R_{L, \mathrm{opt}}$ is obtained by the equation of $R_{L, \mathrm{opt}}=f\left(C_{2}, C_{3}, \ldots, C_{n-1}\right)$. Lastly, $C_{1}$ and $R_{S}$ are chosen to match the imaginary part and real part of input impedance, respectively. The input impedance $\left(Z_{\text {in }}\right)$ is obtained from the equivalent circuit of Figure 2 and is given by

$$
\begin{aligned}
Z_{\text {in }}= & R_{1}+j\left(\omega L_{1}-\frac{1}{\omega C_{1}}\right)+j \omega M_{12}\left(\frac{I_{2}}{I_{1}}\right) \\
& +j \omega M_{13}\left(\frac{I_{3}}{I_{1}}\right)+\cdots+j \omega M_{1, n-1}\left(\frac{I_{n-1}}{I_{1}}\right) \\
& +j \omega M_{1, n}\left(\frac{I_{n}}{I_{1}}\right) .
\end{aligned}
$$

$C_{1, \mathrm{opt}}$ is determined for $\operatorname{Im}\left(Z_{\text {in }}\right)$ to be zero and $R_{S \text {,opt }}$ is set to be $\operatorname{Re}\left(Z_{\text {in }}\right)$ from (5).

\section{Results}

Figure 4 shows the calculated PTE when using the optimum capacitance determined by GA and using the conventional capacitance determined by $C_{i}=1 /\left(\omega_{0}{ }^{2} L_{i}\right)(i=2,3,4)$. Note that $C_{1}$ is given by $\operatorname{Im}\left(Z_{\text {in }}\right)=0$ for impedance matching condition. The structure is shown in Figure 1 when $d_{1}$ and $d_{2}$ scan from 16 to $40 \mathrm{~cm}$ and from 13 to $37 \mathrm{~cm}$, respectively. The PTEs when using the optimum capacitance are much higher and flatter than those using conventional capacitance. The average and standard deviation of PTE are $75.3 \%$ and $5.38 \%$, respectively, when the optimum capacitance is used. On the other hand, when the conventional capacitance [25] is used, the average and standard deviation are $51.8 \%$ and $16.43 \%$, respectively. These results clearly indicate that our method is to find the maximum PTE in the asymmetrical $n$ resonator system, compared with the previous method [25] in the modified paper. The operating frequency of $6.78 \mathrm{MHz}$ is standardized frequency by A4WP for magnetically coupled wireless power transfer system. 


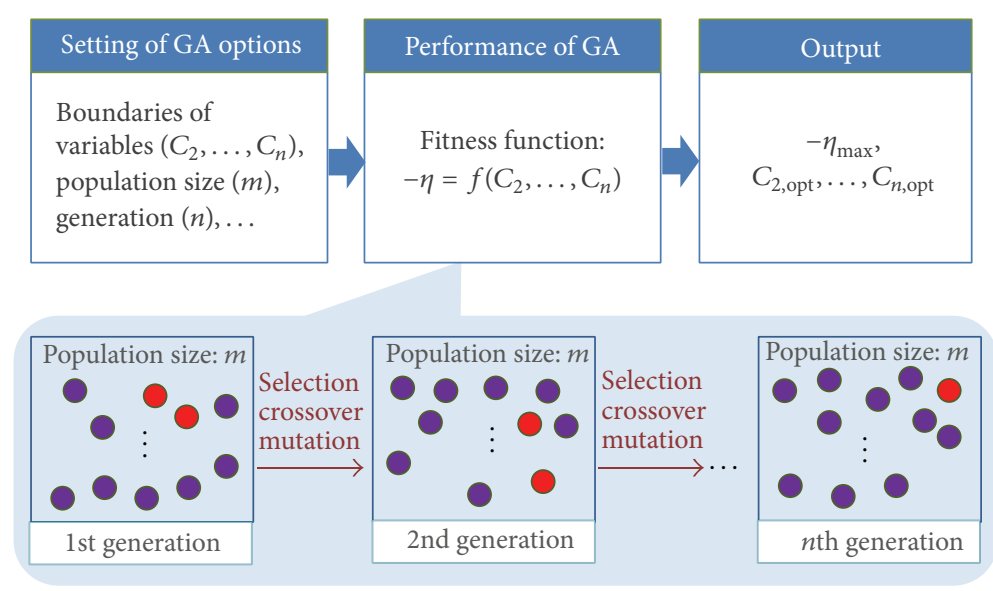

FIgURE 3: Procedure of GA.

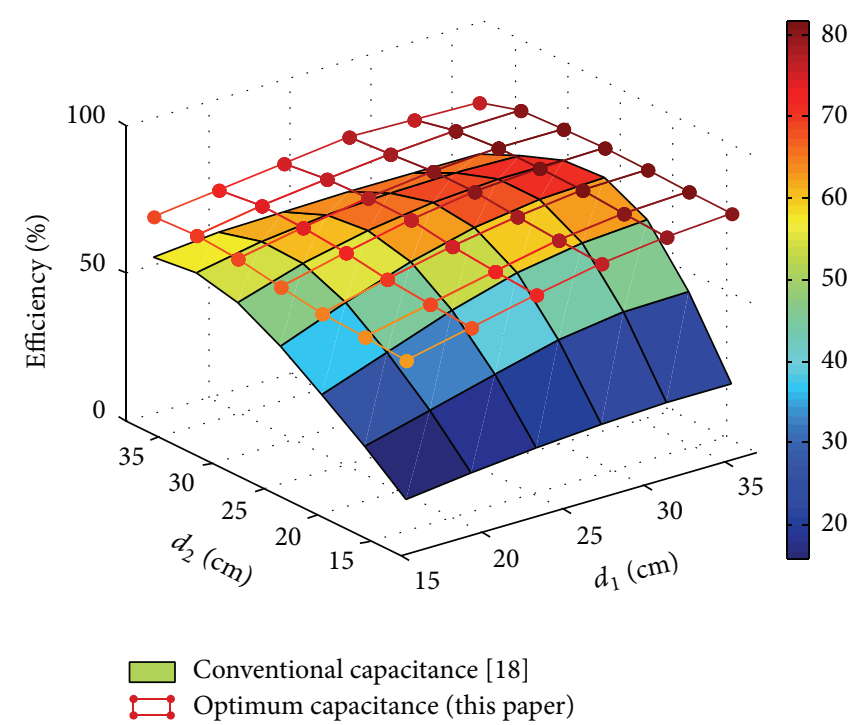

FIGURE 4: Optimum power transfer efficiency versus the various placement of two relay resonators $(f=6.78 \mathrm{MHz})$.

Six cases according to relay position of $d_{1}$ and $d_{2}$ are chosen arbitrarily to demonstrate that our method is generally applied for the various cases. The distances between resonators are shown in Figure 5. Figure 6 shows the photograph of measurement setup. There are four resonators with capacitors in series whose configuration is the case of \#1 in Figure 5. Two feeding loops near resonators 1 and 4 are shown as a source and a load, respectively. The space between resonator 1 and feeding loop (port 1) determines source resistance of $R_{\mathrm{S}}$ and that between feeding loop (port 2 ) and resonator 2 determines load resistance of $R_{L}$ for the impedance matching. Power transfer efficiency (PTE) is given by $\left|s_{21}\right|^{2} /\left(1-\left|s_{11}\right|^{2}\right)$ measured by a vector network analyzer shown in the photograph.

Figure 5 shows the PTEs of six cases, both simulation and measurement. Note that case \#4 is the optimum placement of the relays when $d_{1}$ and $d_{2}$ are found to be $37 \mathrm{~cm}$ and $26 \mathrm{~cm}$, respectively. It is clearly shown that the PTE is improved by using the optimum capacitance at the optimum placement.
The values of $C_{1}, \ldots, C_{4}, R_{S}$, and $R_{L}$ are specified in Table 2 in both cases of optimum capacitance and conventional capacitance. The measured results tend to agree with the simulated results.

However, the efficiencies of measurement are small, compared with the simulation results. The measured errors are thought to be caused by the following reasons. First, the conductivity of the fabricated resonators is lower than that of pure copper used in the simulation. Second, the capacitors have a loss that is not considered in simulation. Lastly, the parameters such as $R_{i}, L_{i}$, and $M_{i j}(i, j=1,2,3,4, i \neq j)$ from HFSS simulator may be slightly different from the fabricated resonator values. It could generate some errors in finding the optimum capacitance value because GA code uses the $R_{i}, L_{i}$, and $M_{i j}$ from HFSS simulator.

Figure 7 shows the calculated dissipation power of four resonators and the load for the six cases when $1 \mathrm{~W}$ power is injected. It is clear that the small dissipated power of four resonators and the large load power indicate the good transfer 


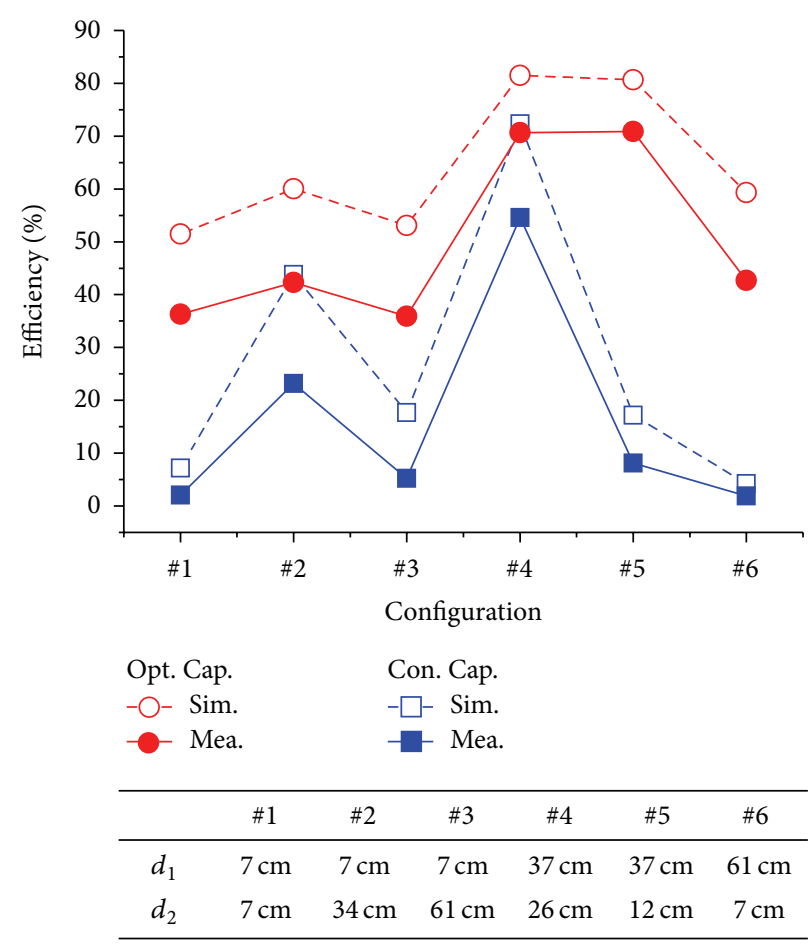

FIgure 5: Power transfer efficiency for six cases.

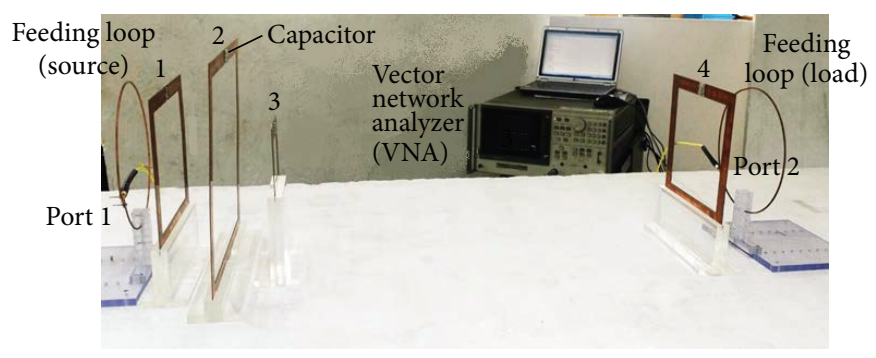

Figure 6: Photograph of experimental setup.

TABLE 1: GA options.

\begin{tabular}{lc}
\hline Classification & Values \\
\hline Variables & $C_{2}, C_{3}, C_{4}$ \\
& $C_{2}: 700 \sim 900 \mathrm{pF}$ \\
Boundary of variables & $C_{3}: 3000 \sim 7000 \mathrm{pF}$ \\
& $C_{4}: 1400 \sim 2000 \mathrm{pF}$ \\
Population & 1000 \\
Generation & 20 \\
Selection & Tournament \\
Crossover & Scattered \\
Mutation & Adaptive Feasible \\
Fitness function & $-\eta=f\left(C_{2}, C_{3}, C_{4}\right)$ \\
\hline
\end{tabular}

efficiency. The power has a relationship to the equation of $P_{i}=(1 / 2)\left|I_{i}\right|^{2} / R_{i}(i=1, \ldots, 4)$ and $P_{L}=(1 / 2)\left|I_{4}\right|^{2} / R_{L}$. Therefore, the dissipated powers are related to the currents on each resonator since $R_{i}(i=1,2,3,4)$ is fixed. In cases of using conventional capacitance, if the distances between two adjacent resonators are longer, the power cannot be transferred well but can be dissipated in the first resonator between them as seen in cases such as cases \#1, \#3, and \#6 in Figure 7. By using optimum capacitance, the currents of resonators can be optimized. For example, the more current is flowing on the large loop of resonator 2 to transfer power more efficiently. Therefore, the more power is to be dissipated in the load. Case \#4 is the most optimized placement $\left(d_{1}\right.$ and $d_{2}$ ) for the maximum PTE in this configuration. By using the optimum capacitance, the PTE is further improved. To be summarized, the optimum capacitance of the asymmetrical relays should be designed to obtain maximum PTE as well as the optimum positions of relay resonators.

\section{Conclusion}

This work presents a new design method of asymmetrical relay resonators for efficient wireless power transfer. The 


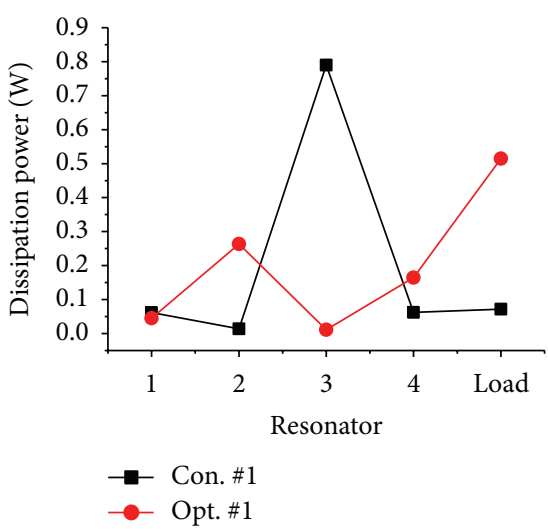

(a)

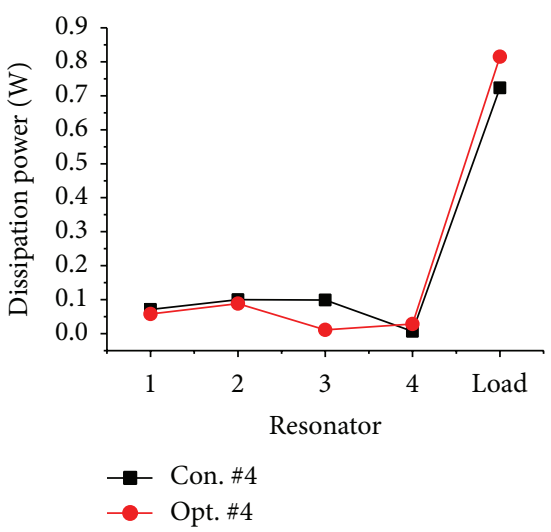

(d)

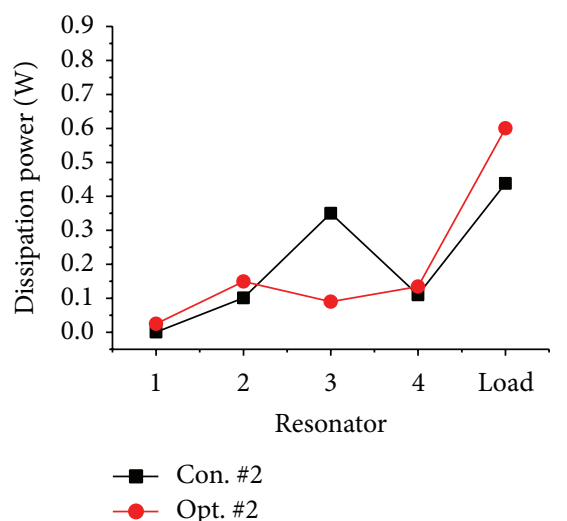

(b)

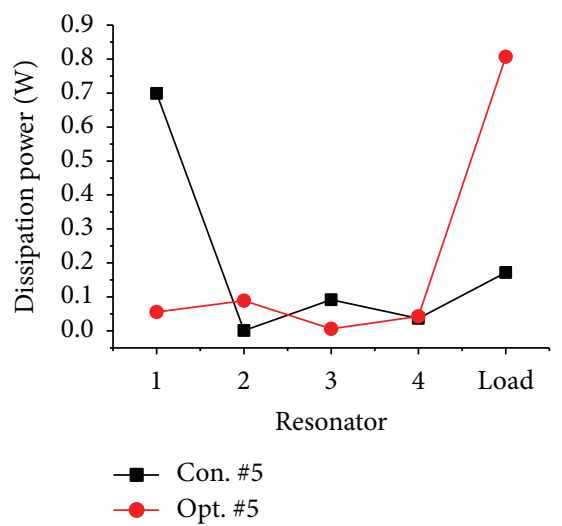

(e)

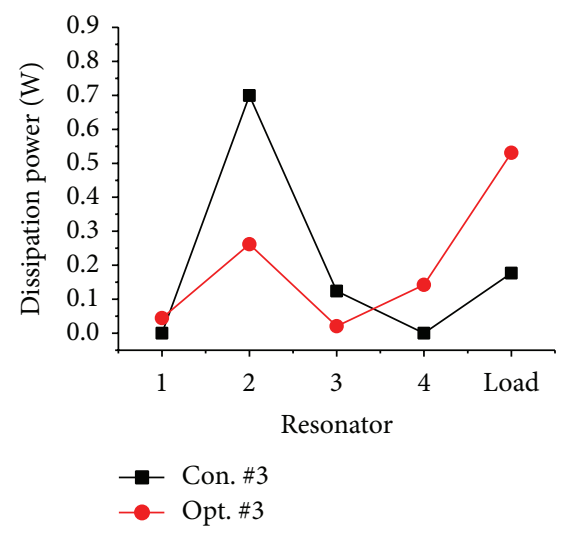

(c)

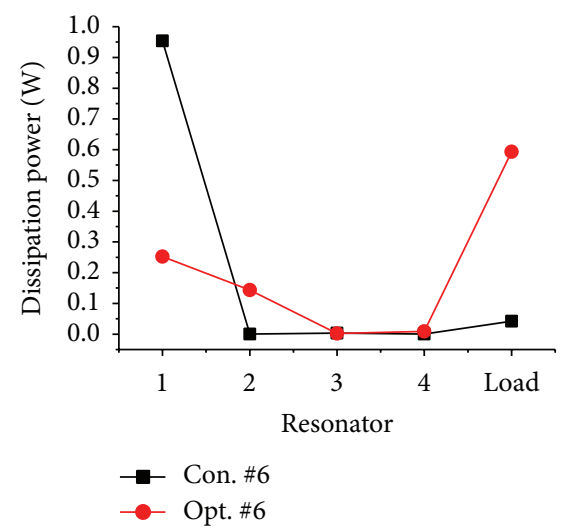

(f)

Figure 7: Dissipated power at four resonators and the load $\left(P_{\text {in }}=1 \mathrm{~W}\right)$.

TABLE 2: Designed parameters: (a) conventional design by $C_{i}=$ $1 /\left(\omega_{0}{ }^{2} L_{i}\right)(i=2,3,4)$ and (b) optimum design by GA.

(a)

\begin{tabular}{|c|c|c|c|c|c|c|}
\hline & $\# 1$ & $\# 2$ & $\# 3$ & $\# 4$ & $\# 5$ & $\# 6$ \\
\hline$R_{L}(\Omega)$ & 0.021 & 0.071 & 54.26 & 1.97 & 0.083 & 3.99 \\
\hline$R_{S}(\Omega)$ & 0.289 & 42.37 & 383.59 & 0.252 & 0.026 & 0.019 \\
\hline$C_{1}(\mathrm{pF})$ & 1841 & 618.5 & 836 & 1483 & 1496 & 1495 \\
\hline$C_{2}(\mathrm{pF})$ & \multicolumn{6}{|c|}{821.6} \\
\hline$C_{3}(\mathrm{pF})$ & \multicolumn{6}{|c|}{5018} \\
\hline$C_{4}(\mathrm{pF})$ & \multicolumn{6}{|c|}{1495} \\
\hline
\end{tabular}

(b)

\begin{tabular}{lcccccc}
\hline & $\# 1$ & $\# 2$ & $\# 3$ & $\# 4$ & $\# 5$ & $\# 6$ \\
\hline$R_{L}(\Omega)$ & 0.056 & 0.08 & 0.067 & 0.518 & 0.335 & 1.165 \\
$R_{S}(\Omega)$ & 0.397 & 0.717 & 0.401 & 0.311 & 0.323 & 0.07 \\
$C_{1}(\mathrm{pF})$ & 1001 & 1004 & 1008 & 1494 & 1494 & 1495 \\
$C_{2}(\mathrm{pF})$ & 758 & 764 & 765 & 819 & 813 & 800 \\
$C_{3}(\mathrm{pF})$ & 3405 & 4960 & 3878 & 4597 & 4151 & 2584 \\
$C_{4}(\mathrm{pF})$ & 1495 & 1495 & 1454 & 1467 & 1493 & 1069 \\
\hline
\end{tabular}

optimum design of asymmetrical relay is performed by both optimum distances between the relays and optimum capacitance of resonators. The optimum locations of the asymmetrical relay resonators were found as the positions of the relays are scanned. To further improve the PTE, the optimum capacitance of the resonators was determined by GA. To find optimum capacitance, GA can replace the complicated simultaneous equations. The PTEs when using the optimum capacitance are much higher and flatter than those using conventional capacitance as the positions of the relays are scanned. $C_{1}$ and $R_{S}$ are not related to the PTE but are related to the impedance matching. The dissipated powers of each resonator and load are investigated, which assures that optimum currents of resonators are set by the optimum capacitance to transfer power more efficiently. The design method can be extended and applied to different configurations, that is, the relay resonators including a source and a load.

\section{Competing Interests}

The authors declare that they have no competing interests.

\section{Acknowledgments}

This research was supported by Basic Science Research Program through the National Research Foundation of 
Korea (NRF) funded by the Ministry of Education (no. 2015R1A6A1A03031833).

\section{References}

[1] J. Jadidian and D. Katabi, "Magnetic MIMO: how to charge your phone in your pocket," in Proceedings of the 20th ACM Annual International Conference on Mobile Computing and Networking (MobiCom '14), pp. 495-506, Maui, Hawaii, USA, September 2014.

[2] S. Y. Choi, B. W. Gu, S. Y. Jeong, and C. T. Rim, "Advances in wireless power transfer systems for roadway-powered electric vehicles," IEEE Journal of Emerging and Selected Topics in Power Electronics, vol. 3, no. 1, pp. 18-36, 2015.

[3] B. H. Waters, A. P. Sample, P. Bonde, and J. R. Smith, "Powering a ventricular assist device (VAD) with the free-range resonant electrical energy delivery (FREE-D) system," Proceedings of the IEEE, vol. 100, no. 1, pp. 138-149, 2012.

[4] M. A. Adeeb, A. B. Islam, M. R. Haider, F. S. Tulip, M. N. Ericson, and S. K. Islam, "An inductive link-based wireless power transfer system for biomedical applications," Active and Passive Electronic Components, vol. 2012, Article ID 879294, 11 pages, 2012.

[5] G. Pan and L. Wang, "Swallowable wireless capsule endoscopy: progress and technical challenges," Gastroenterology Research and Practice, vol. 2012, Article ID 841691, 9 pages, 2012.

[6] K. Na, H. Jang, H. Ma, and F. Bien, "Tracking optimal efficiency of magnetic resonance wireless power transfer system for biomedical capsule endoscopy," IEEE Transactions on Microwave Theory and Techniques, vol. 63, no. 1, pp. 295-304, 2015.

[7] 2016, http://a4wppmamerge.com/.

[8] A. Kurs, A. Karalis, R. Moffatt, J. D. Joannopoulos, P. Fisher, and M. Soljačić, "Wireless power transfer via strongly coupled magnetic resonances," Science, vol. 317, no. 5834, pp. 83-86, 2007.

[9] A. P. Sample, D. A. Meyer, and J. R. Smith, "Analysis, experimental results, and range adaptation of magnetically coupled resonators for wireless power transfer," IEEE Transactions on Industrial Electronics, vol. 58, no. 2, pp. 544-554, 2011.

[10] Z. Dang, Y. Cao, and J. A. A. Qahouq, "Reconfigurable magnetic resonance-coupled wireless power transfer system," IEEE Transactions on Power Electronics, vol. 30, no. 11, pp. 6057-6069, 2015.

[11] G. Kim, T.-K. Oh, and B. Lee, "Effects of metamaterial slabs applied to wireless power transfer at $13.56 \mathrm{MHz}$," International Journal of Antennas and Propagation, vol. 2015, Article ID 840135, 7 pages, 2015.

[12] J. Park, Y. Tak, Y. Kim, Y. Kim, and S. Nam, "Investigation of adaptive matching methods for near-field wireless power transfer," IEEE Transactions on Antennas and Propagation, vol. 59, no. 5, pp. 1769-1773, 2011.

[13] T. P. Duong and J.-W. Lee, "Experimental results of highefficiency resonant coupling wireless power transfer using a variable coupling method," IEEE Microwave and Wireless Components Letters, vol. 21, no. 8, pp. 442-444, 2011.

[14] S. Lu, C. Zuo, and C. Piao, "The parameters optimization of MCR-WPT system based on the improved genetic simulated annealing algorithm," Mathematical Problems in Engineering, vol. 2015, Article ID 174868, 10 pages, 2015.
[15] Q. Zhu, Y. Guo, L. Wang, C. Liao, and F. Li, "Improving the misalignment tolerance of wireless charging system by optimizing the compensate capacitor," IEEE Transactions on Industrial Electronics, vol. 62, no. 8, pp. 4832-4836, 2015.

[16] F. Zhang, S. A. Hackworth, W. Fu, C. Li, Z. Mao, and M. Sun, "Relay effect of wireless power transfer using strongly coupled magnetic resonances," IEEE Transactions on Magnetics, vol. 47, no. 5, pp. 1478-1481, 2011.

[17] X. Zhang, S. L. Ho, and W. N. Fu, "Quantitative design and analysis of relay resonators in wireless power transfer system," IEEE Transactions on Magnetics, vol. 48, no. 11, pp. 4026-4029, 2012.

[18] D. Ahn and S. Hong, "A study on magnetic field repeater in wireless power transfer," IEEE Transactions on Industrial Electronics, vol. 60, no. 1, pp. 360-371, 2012.

[19] B.-C. Park, Y.-H. Son, B.-J. Jang, and J. H. Lee, "Realization of alignment-free WPT system," Journal of Electromagnetic Engineering and Science, vol. 14, no. 4, pp. 329-331, 2014.

[20] W. Zhong, C. K. Lee, and S. Y. Ron Hui, "General analysis on the use of Tesla's resonators in domino forms for wireless power transfer," IEEE Transactions on Industrial Electronics, vol. 60, no. 1, pp. 261-270, 2013.

[21] G. Lee, B. H. Waters, C. Shi, W. S. Park, and J. R. Smith, “Design considerations for asymmetric magnetically coupled resonators used in wireless power transfer applications," in Proceedings of the IEEE Topical Meeting on Biomedical Wireless Technologies Networks and Sensing Systems, pp. 20-23, Austin, Tex, USA, January 2013.

[22] Y. Zhang, T. Lu, Z. Zhao, K. Chen, F. He, and L. Yuan, "Wireless power transfer to multiple loads over various distances using relay resonators," IEEE Microwave and Wireless Components Letters, vol. 25, no. 5, pp. 337-339, 2015.

[23] C. K. Lee, W. X. Zhong, and S. Y. R. Hui, "Effects of magnetic coupling of nonadjacent resonators on wireless power dominoresonator systems," IEEE Transactions on Power Electronics, vol. 27, no. 4, pp. 1905-1916, 2012.

[24] J.-H. Kim, B.-C. Park, and J.-H. Lee, "Optimum design of Wpt relay system by controlling capacitance," Microwave and Optical Technology Letters, vol. 56, no. 7, pp. 1658-1661, 2014.

[25] J.-H. Kim, B.-C. Park, and J.-H. Lee, "New analysis method for wireless power transfer system with multiple n resonators," Journal of Electromagnetic Engineering and Science, vol. 13, no. 3, pp. 173-177, 2013.

[26] A. G. Lazaropoulos, "Practical coupled resonators in domino arrangements for power transmission and distribution: replacing step-down power transformers and their branches across the power grid," Journal of Energy, vol. 2013, Article ID 795835, 25 pages, 2013.

[27] S. Y. R. Hui, W. Zhong, and C. K. Lee, "A critical review of recent progress in mid-range wireless power transfer," IEEE Transactions on Power Electronics, vol. 29, no. 9, pp. 4512-4520, 2014.

[28] 2016, https://en.wikipedia.org/wiki/Genetic_algorithm, http:// www.mathworks.com/discovery/genetic-algorithm.html?s_tid =gn_loc_drop. 


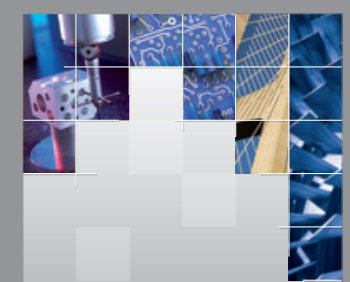

\section{Enfincering}
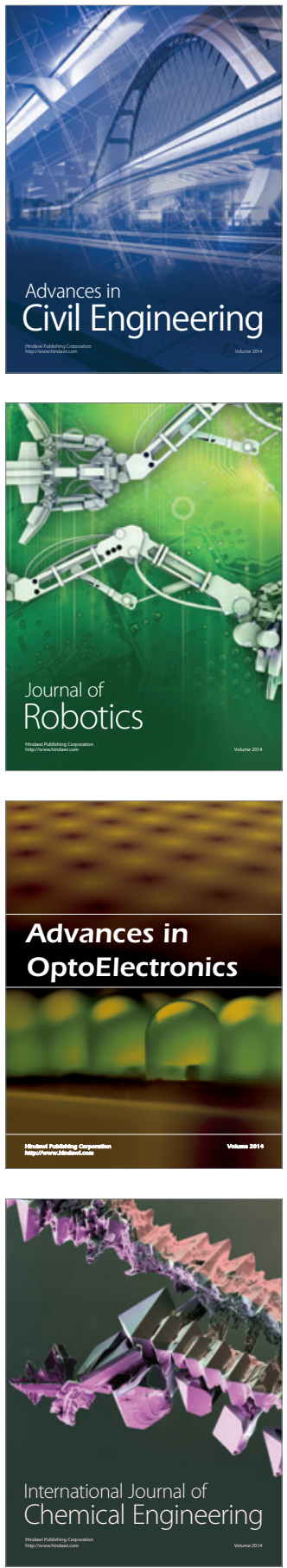

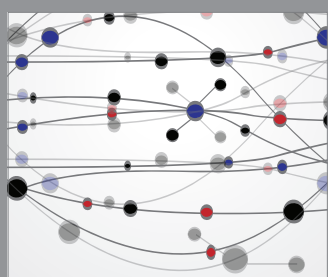

The Scientific World Journal

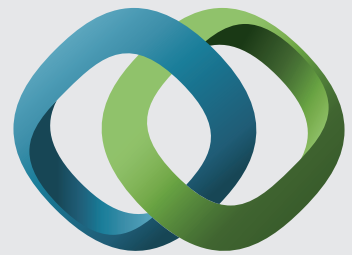

\section{Hindawi}

Submit your manuscripts at

http://www.hindawi.com
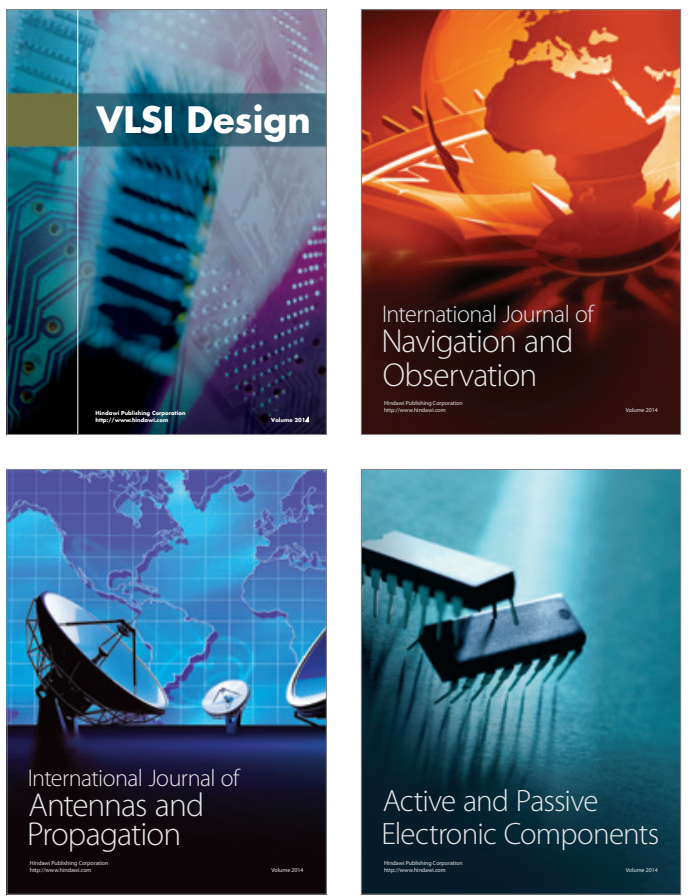
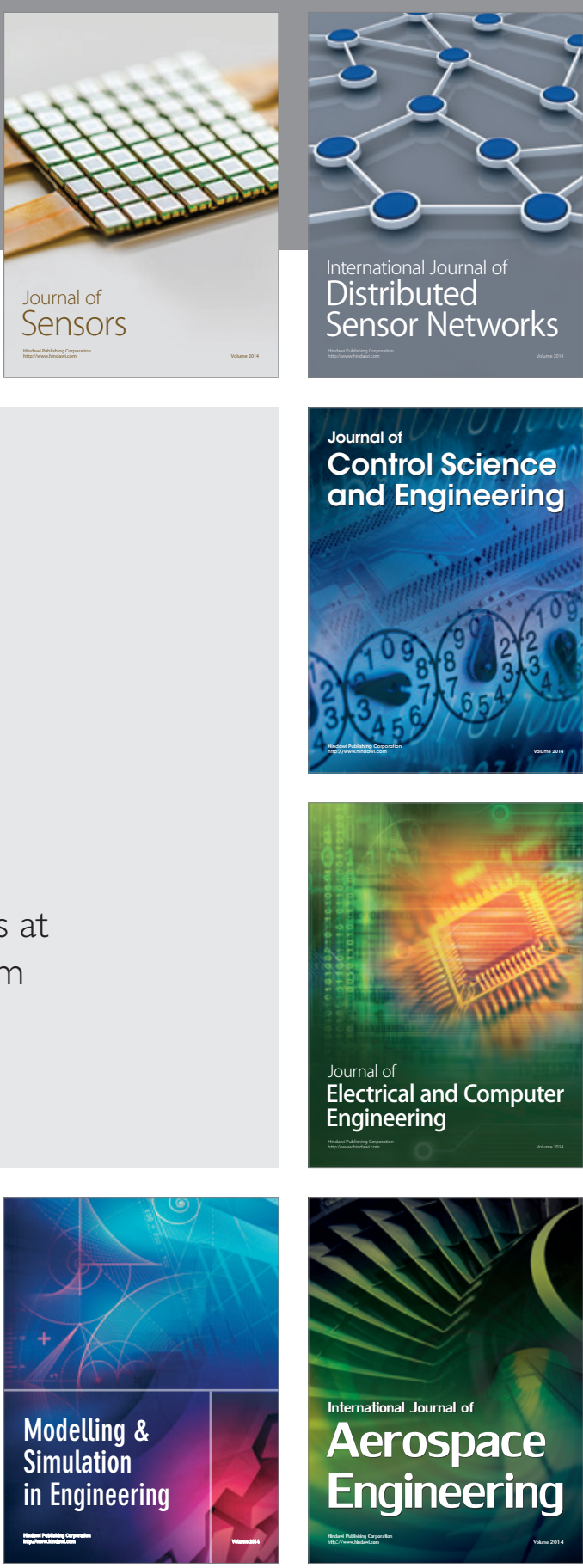

International Journal of

Distributed

Sensor Networks

Journal of

Control Science

and Engineering
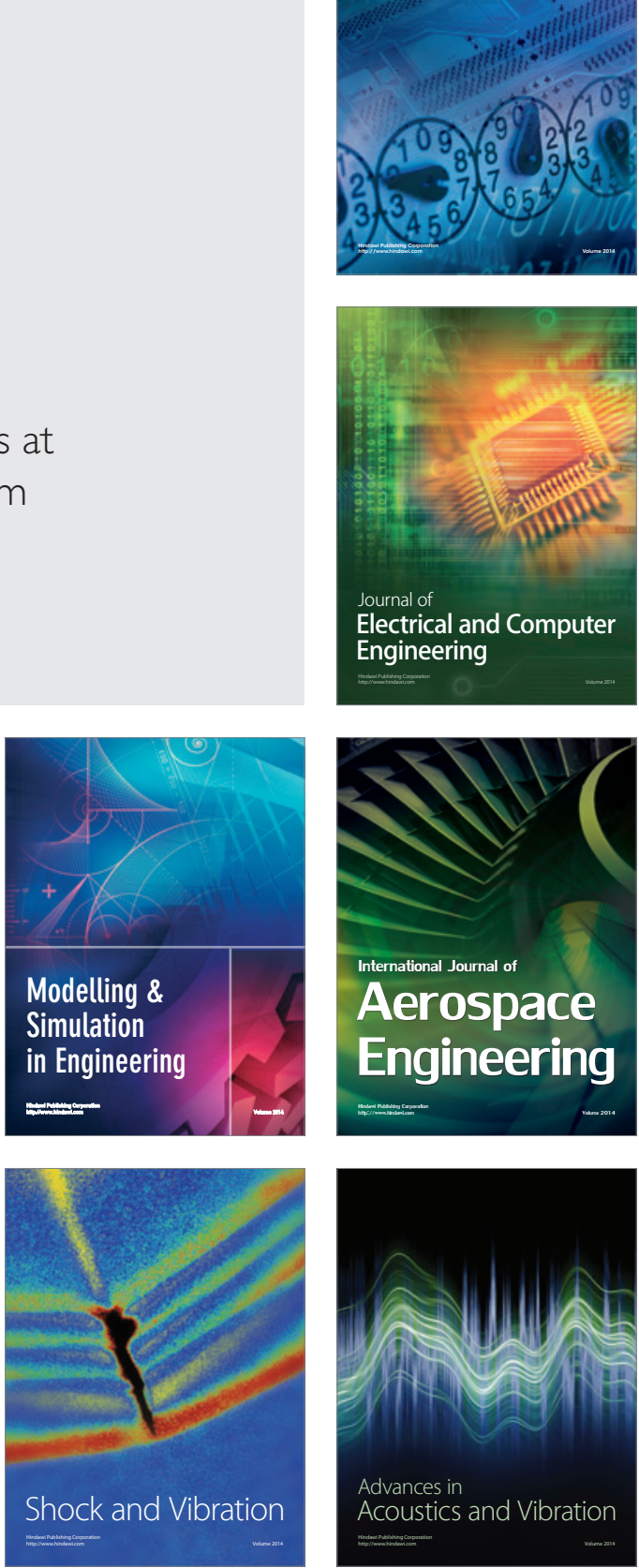